\title{
Cell-Conditioned Protein Coronas on
}

\section{Engineered Particles Influence Immune}

\section{Responses}

Qiong Dai, ${ }^{\dagger}$ Junling Guo, ${ }^{\dagger}$ Yan Yan, ${ }^{\ddagger}$ Ching-Seng Ang, ${ }^{\S}$ Nadja Bertleff-Zieschang, ${ }^{\dagger}$ and

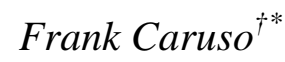

\begin{abstract}
${ }^{\dagger}$ ARC Centre of Excellence in Convergent Bio-Nano Science and Technology, and the Department of Chemical and Biomolecular Engineering, The University of Melbourne, Parkville, Victoria 3010, Australia; ${ }^{\ddagger}$ Centre for BioNano Interactions, School of Chemistry and Chemical Biology, University College Dublin, Dublin, Ireland; and ${ }^{\S}$ Bio21 Molecular Science and Biotechnology Institute, The University of Melbourne, Parkville, Victoria 3010, Australia.
\end{abstract}

\begin{abstract}
A protein corona, which forms on engineered particles as soon as they are introduced into biological environments, is known to provide particles with a 'biological identity’. Protein coronas derived from various biological environments have been demonstrated to alter the cell internalization mechanism, to diminish targeting ability and to induce nanoparticle aggregation. So far, most of these studies have challenged engineered particles with a static biological environment. However, the extracellular environment is highly dynamic due to the process termed 'cell conditioning', in which
\end{abstract}


cells deplete and secrete biomolecules. In this work, we demonstrate that protein coronas formed on engineered particles from such cell-conditioned media affect the biophysical particle properties and protein adsorption differently to protein coronas from an unconditioned environment. When investigating particles with protein coronas formed in various biologically relevant environments toward their interaction with immune cells we observed differences in pro-inflammatory cytokine secretion and immune cell apoptosis. Interestingly, we found that the particles either increased or mitigated the secretion of a specific cytokine, depending on the environment where the protein corona was formed. Our study suggests that the use of protein coronas could be useful to engineer drug carriers for elongated circulation, enhanced biocompatibility, and lower toxicity by triggering a specific immune response.

KEYWORDS: protein corona, cell conditioning, immune response, cytokine, apoptosis, cell uptake

\section{INTRODUCTION}

When nanoengineered particles designed for biological applications enter a physiological environment they are surrounded by a biological fluid of complex composition including proteins, lipids, metabolites and ions. It is widely accepted that proteins and biomolecules from this environment adsorb on the particle surface and form a 'protein corona' (PC),

which imparts the chemically designed particle with a 'biological identity'. ${ }^{1-3}$ Highly 
abundant proteins bind rapidly to the surface and are eventually replaced by less abundant proteins that show a higher affinity for the particle surface. ${ }^{4-7}$ In equilibrium the inner near-monolayer corona is termed the 'hard' PC (hPC), in which molecules bind tightly but not completely irreversibly. ${ }^{1}$ The outer layer is referred to as the 'soft' PC (sPC) and is formed by more loosely associated and more rapidly exchanging proteins. ${ }^{1}$ It has been demonstrated that the formation of PCs depends on both the biological environment and the physicochemical properties of particles, such as size, curvature, hydrophobicity, charge, and surface functionality. ${ }^{8-12}$ While the SPC is highly dynamic and 'evolves' with time, ${ }^{13,14}$ in particular when particles are transferred from one biological environment to another, the hPC derived from a previous environment is partially retained. ${ }^{2,13-15}$ Therefore, PCs, especially hPCs, can provide a 'fingerprint' and information on environments to which particles have been exposed to. ${ }^{16}$

Previous studies have shown that the PC plays a key role in particle-cell recognition and ensuing interactions. ${ }^{1}$ For example, it has been reported that PCs reduce the cellular uptake of nanoparticles through the inhibition of cell membrane adhesion ${ }^{17,18}$ and that the presence of a PC can dramatically decrease or totally eliminate the targeting ability by shielding the targeting ligand tethered to the particle surface. ${ }^{19-21}$ Moreover, it has been shown that PCs affect the internalization mechanisms of particles by changing the dominant internalization route. ${ }^{22}$ In addition, the adsorbed proteins might unfold and initiate different cell signaling pathways or cause unexpected inflammatory responses. ${ }^{3,23,24}$ For example, the same type of PC derived from fetal bovine serum (FBS) has been reported to play different roles in the uptake of polymeric particles by immune cells. ${ }^{3}$ Upon adsorption of BSA on the surface of the polymeric particles the protein 
underwent conformational changes, leading to lower internalization efficiency by monocytic THP-1 cells. In contrast, Class A Scavenger Receptor (SR-A) expressed on the surface of macrophage-like dTHP-1 cells recognized denatured BSA in the proteinparticle complex and initiated an internalization of the BSA-particle complex via SR-Amediated phagocytosis. ${ }^{3}$

So far the majority of studies investigating the effect of PCs on particle-cell interactions have used 'static' sources of PCs, including albumin, fibrinogen, FBS, human serum (HS) and human plasma (HP). ${ }^{3,9,19,23}$ However, these environments do not fully reflect the highly dynamic nature of extracellular environments. ${ }^{25,26}$ It is known that living cells continuously internalize nutrients from the environment for normal cell function, growth and proliferation, and secrete products into the environment. ${ }^{25,26}$ This process, known as cell conditioning, continually alters the components of extracellular environments, including proteins, nutrients, and ions. ${ }^{25-27}$ For example, it was shown that incubation of gold nanoparticles (AuNPs) in various cell-conditioned environments leads to PCs of different compositions and induces AuNP aggregation. ${ }^{27}$ Interestingly, NP aggregation was not only observed for the whole conditioned media, but also for its protein-free filtrate, indicating the involvement of low molecular weight components (e.g. biomolecules, nutrients, ions) in this process. ${ }^{27}$ AuNPs with cell-conditioned PCs showed higher cell affinity, uptake, and retention of the AuNPs, presumably due to the different biological identity of AuNP aggregates. ${ }^{27}$ While this study highlighted the influence of cell-conditioned media on the PC of particles, its implications on subsequent immune responses upon interactions between immune cells and cell-conditioned PC-coated particles have not been reported. Since the interaction of particles with the immune 
system is one of the key factors that determines the in vivo circulation of particles, ${ }^{28}$ it is of importance to study how PCs, especially cell-conditioned PCs, affect particle-immune cell interactions.

Herein, we formed PCs on the surface of multilayered poly(methacrylic acid) (PMA) particles under various unconditioned and cell-conditioned environments, and compared the influence of the PCs on the surface properties of the particles, the particle-cell interactions, and the particle-induced immune responses, such as cytokine secretion and cell death/apoptosis (Scheme 1). We have previously developed PMA particles, generated via layer-by-layer (LbL) assembly, for various biological applications, and have shown their potential for drug delivery, ${ }^{29}$ vaccine delivery, ${ }^{30}$ and as microreactors. ${ }^{31}$ In this study, we examined the formation and impact of PCs on both hollow capsules (CAPs) and core-shell particles (CSPs) because they are different in rigidity due to the absence or presence of a silica core, which has resulted in different in vitro and in vivo outcomes. ${ }^{32}$ By exposing CAPs and CSPs to FBS, HS, HP, or media from different cell cultures, various unconditioned and cell-conditioned PCs were formed. These PCs differed in protein composition, influenced the surface properties of particles, and affected cytokine secretion and apoptosis levels upon particle-immune cell interactions. We found that these changes depended on 1) the properties of the particles (CAPs vs. CSPs), 2) the immune cell line (macrophage-like vs. monocyte) and 3) the biological environment for PC formation, and more specifically, the cell phenotype that was used for 'conditioning' of the medium. Importantly, the level of a particular cytokine (e.g. IL$1 \beta$, MCP-1) could be both increased and decreased by a different PC on the same particle system, suggesting that the rational design of PCs can offer an alternative strategy for 
particle surface functionalization. Such a biomimetic functionalization approach could potentially achieve specific physiological objectives, including elongated circulation, enhanced targeting outcomes, lower toxicity, and improved vaccine delivery. 


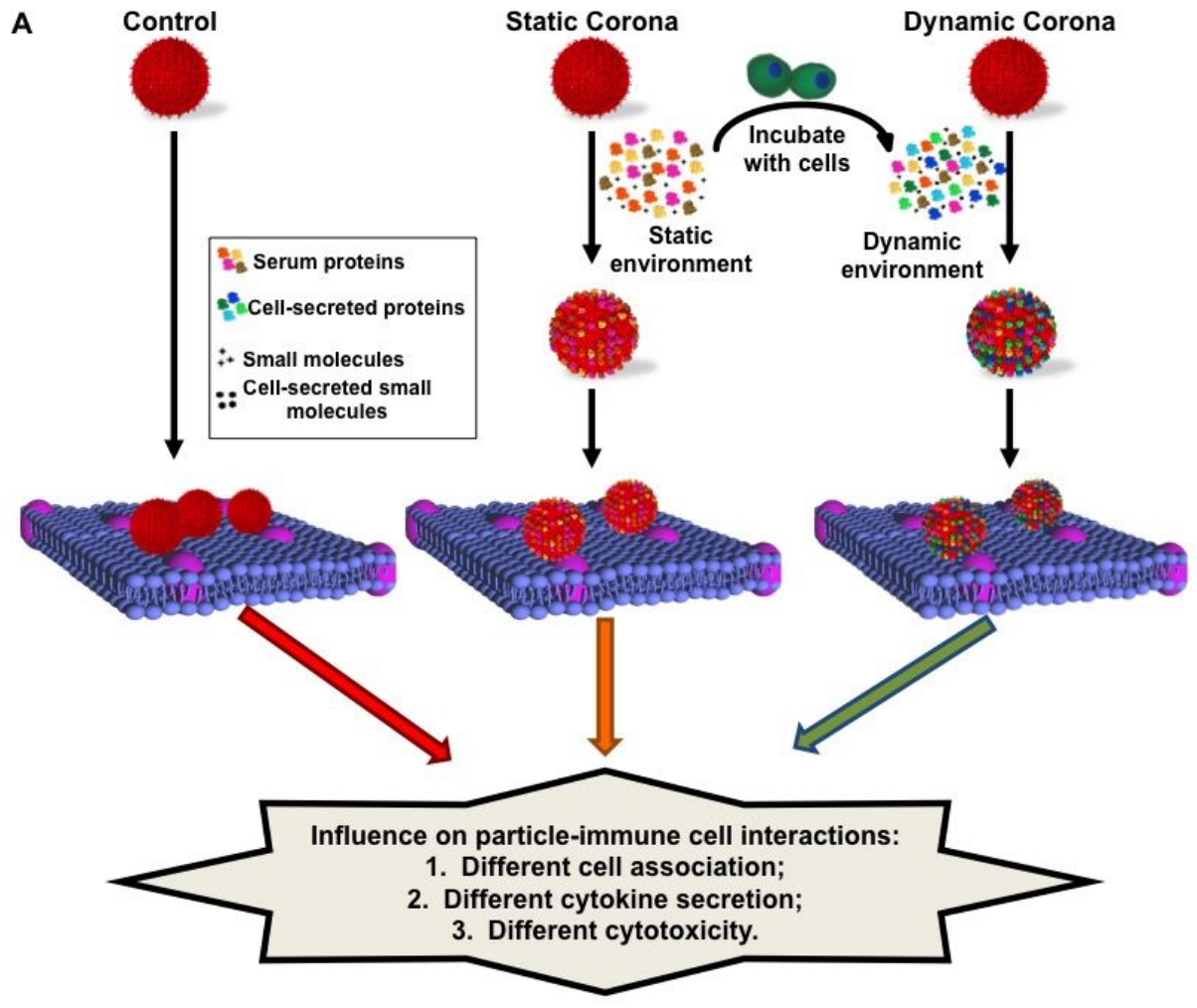

B

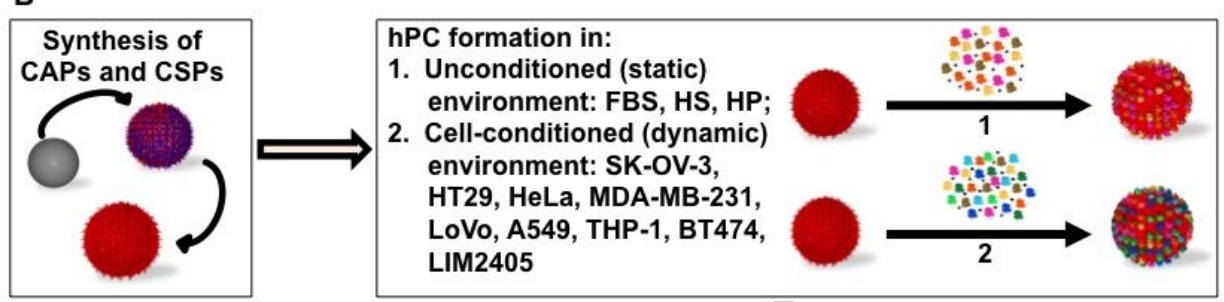
Analysis of hPCcoated particles

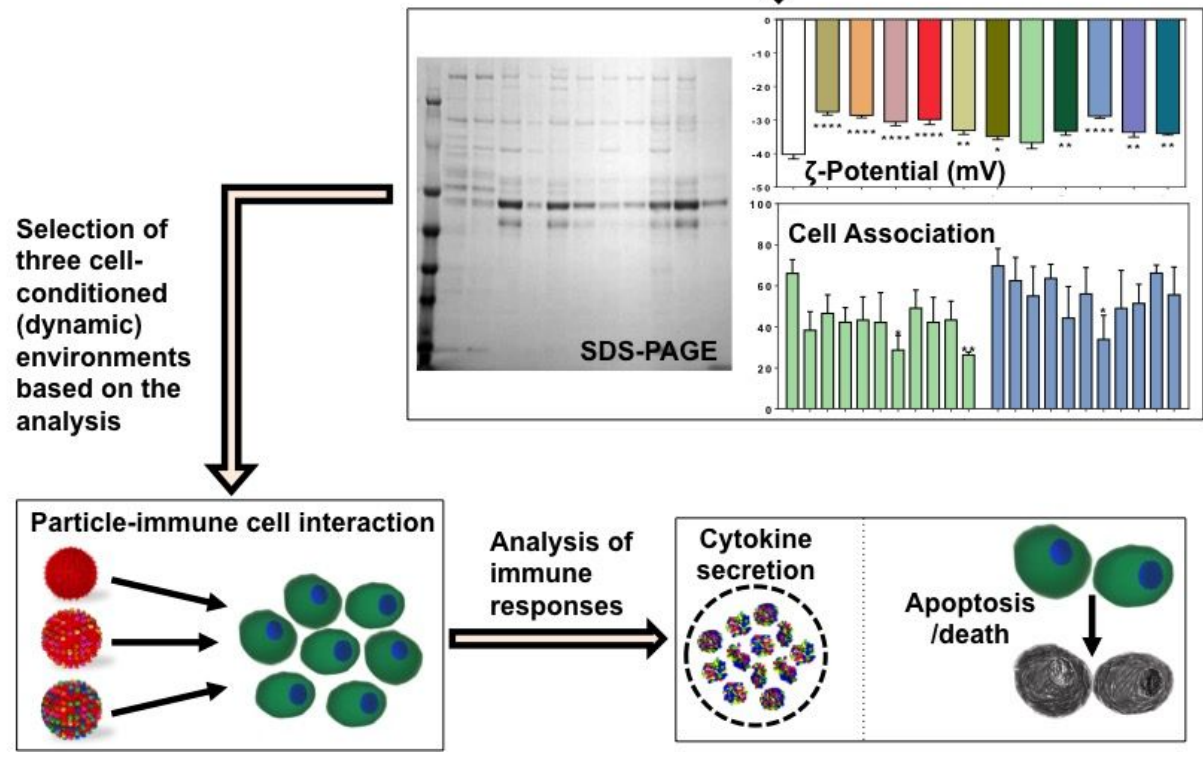


Scheme 1. A) Schematic illustration of the formation of PCs on PMA particles in unconditioned or cell-conditioned environments, and the subsequent interaction between cells and non-coated or protein-coated particles. The biological environment contains small molecules such as metabolites, nutrients and ions (e.g. glucose, triglycerides, cholesterol) that are internalized and secreted by cells in the process of cell-conditioning. B) Workflow scheme of the experimental design to investigate particle-immune cell interactions. After particle synthesis, hPCs from various unconditioned and cellconditioned environments were formed and analyzed for protein adsorption, $\zeta$-potential and cell association. Based on these findings, hPCs from three cell-conditioned environments (LoVo, HeLA, THP-1) were compared with hPCs from an unconditioned environment (FBS, HS, HP) with respect to their effect on immune responses.

\section{EXPERIMENTAL SECTION}

Materials. Poly(methacrylic acid) (PMA, Mw $\sim 15 \mathrm{kDa}$ ) was purchased from Polysciences (Warrington, PA, USA). $\mathrm{SiO}_{2}$ particles were purchased from microParticles GmbH (Berlin, Germany). Hydrofluoric acid (HF), ammonium fluoride $\left(\mathrm{NH}_{4} \mathrm{~F}\right)$, sodium acetate (NaOAc), poly( $N$-vinylpyrrolidone) (PVPON; $M w \sim 10 \mathrm{kDa}$ ), sodium ascorbate, copper(II) sulfate $\left(\mathrm{CuSO}_{4}\right)$, phosphate-buffered saline (PBS) tablets, 1-ethyl-3-(3(dimethylamino)propyl) carbodiimide (EDC), dimethyl sulfoxide (DMSO), propargylamine hydrochloride, deuterium oxide, phorbol 12-myristate 13-acetate (TPA), 2.5\% trypsin solution, and 4-(4,6-dimethoxy-1,3,5-triazin-2-yl)-4-methylmorpholin-4ium (DMTMM) were purchased from Sigma Aldrich (St. Louis, MO, USA) and used as

received. AF633 reactive dye, Roswell Park Memorial Institute 1640 (RPMI1640), 
Kaighn's Modification of Ham's F-12 Medium (F-12K), and Dulbecco's Modified Eagle Medium (DMEM) media containing L-glutamine (300 $\left.\mathrm{mg} \mathrm{L}^{-1}\right)$, fetal bovine serum (FBS), Dulbecco phosphate-buffered saline (DPBS), NuPAGE Bis-Tris precast gel 4-12\%, NuPAGE MOPS SDS running buffer, NuPAGE LDS sample buffer, NuPAGE sample reducing agent, Chromatin Condensation \& Membrane Permeability Dead Cell Apoptosis Kit with Hoechst 33342, YO-PRO®-1, and propidium iodide (PI) dyes, for flow cytometry, and Alexa Fluor 488-conjugated wheat germ agglutinin (WGA488) were purchased from Life Technologies (Grand Island, NY, USA). Human Serum (HS) and Human Plasma (HP) were obtained from BioreclamationIVT. Paraformaldehyde (4\%) was purchased from Electron Microscopy Sciences (Hatfield, PA, USA). Ultrapure water with a resistance greater than $18 \mathrm{M} \Omega \mathrm{cm}$ was obtained from an inline Millipore RiOs/Origin system (Millipore Corporation, MA, USA). Enzyme-linked immunosorbent assay (ELISA) kits were purchased from ELISAKIT.COM PTY LTD(Scoresby, VIC, Australia).

LbL Particle Preparation. The LbL particles were fabricated as described previously. ${ }^{8,19}$ The synthesis of alkyne-functionalized poly(methacrylic acid) (PMA Alk; $_{\text {; }}$ alkyne functionalization degree $\sim 8 \%$ ) is described in the Supporting Information. The synthesis of the disulfide reducible cross-linker (click cross-linker), $N, N^{\prime}$-(dithiodiethane2,1-diyl)bis(1-azido tetraethylene glycol acetamide) and subsequent LbL assembly of the degradable CAPs and CSPs were outlined previously. ${ }^{8,33}$ A standard washing procedure was employed as follows: a $200 \mu \mathrm{L} \mathrm{SiO}_{2}$ particle suspension (50 mg mL ${ }^{-1}$; diameter 519 $\mathrm{nm}$ ) was centrifuged at $1000 \mathrm{~g}$ for $90 \mathrm{~s}$. The supernatant was removed and the particles were dispersed in $1000 \mu \mathrm{L}$ of NaOAc buffer $(\mathrm{pH} 4, \quad 50 \mathrm{mM})$. Three 
centrifugation/redispersion cycles were conducted. Following washing, $1000 \mu \mathrm{L}$ of PVPON (1 mg mL $\left.{ }^{-1}\right)$ in NaOAc buffer ( $\mathrm{pH} \mathrm{4,50} \mathrm{mM)} \mathrm{was} \mathrm{added} \mathrm{to} \mathrm{the} \mathrm{particles} \mathrm{(for}$ adsorption onto the silica surface) for 15 min with constant shaking. The resulting particles were washed using the standard washing procedure described above (three centrifugation/redispersion cycles using NaOAc buffer). Then, to adsorb a PMA $\mathrm{Alk}_{\text {lk }}$ layer onto the PVPON layer, $1000 \mu \mathrm{L}$ of PMA $\mathrm{Plk}_{\text {Alk }}\left(1 \mathrm{mg} \mathrm{mL}^{-1}\right)$ prepared in NaOAc buffer (pH 4, $50 \mathrm{mM}$ ) was used with constant shaking for $15 \mathrm{~min}$. The particles were then washed to create a PVPON/PMA $A_{\text {Alk }}$ bilayer. The PVPON/PMA $A_{\text {Alk }}$ bilayer adsorption process was repeated four more times. The multilayer shell was covalently cross-linked by incubating the particles with a $1500 \mu \mathrm{L}$ solution containing the click cross-linker $\left(1 \mathrm{mg} \mathrm{mL}^{-1}\right)$, sodium ascorbate $\left(4.4 \mathrm{mg} \mathrm{mL}^{-1}\right)$, and $\mathrm{CuSO}_{4}\left(1.8 \mathrm{mg} \mathrm{mL}^{-1}\right)$ at a v/v/v ratio of 3:1:1 in NaOAc buffer ( $\mathrm{pH}$ 4, $50 \mathrm{mM}$ ) for $12 \mathrm{~h}$ with constant shaking. The cross-linked particles were then washed three times with NaOAc buffer ( $\mathrm{pH}$ 4, $50 \mathrm{mM}$ ).

The CAPs were formed by dissolving the silica templates using $\mathrm{NH}_{4} \mathrm{~F}$ (13.3 M)buffered HF (5 M) at $\mathrm{pH}$ 4. (Caution! HF is highly toxic and great care must be taken during handling). The CAPs were centrifuged (4200 g, $7 \mathrm{~min}$ ) and washed three times

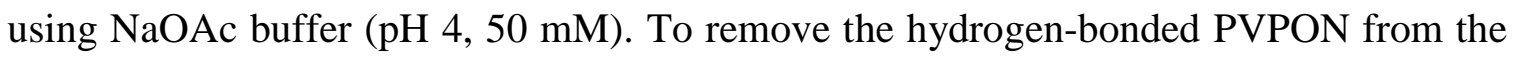
cross-linked multilayers, the CAPs or CSPs were incubated in PBS buffer at pH 7.4 for approximately $12 \mathrm{~h}$.

Fluorescence Labeling of Particles. The particles were labeled with AF633, as described previously. ${ }^{8}$ First, $500 \mu \mathrm{L}$ of EDC $\left(10 \mathrm{mg} \mathrm{mL}^{-1}\right)$ prepared in PBS buffer at $\mathrm{pH}$ 7.4 was mixed with $500 \mu \mathrm{L}$ of five-layered PMA CaP or CSP suspensions in PBS. Subsequently, $5 \mu \mathrm{L}$ of AF633 $\left(1 \mathrm{mg} \mathrm{mL}^{-1}\right)$ in dry DMSO was added to the mixture. 
Samples were incubated in the dark with constant shaking for $16 \mathrm{~h}$ at $\sim 23{ }^{\circ} \mathrm{C}$. After labeling, the CAPs and CSPs were washed three times in PBS and counted using flow cytometry (Apogee Flow) to determine their concentration.

Formation of Protein Corona-Coated Particles. PMA CAPs or CSPs $\left(1 \times 10^{8}\right)$ were incubated in $500 \mu \mathrm{L}$ of protein-containing media (FBS, HS, HP, or various media from cell culture) for $1 \mathrm{~h}$ at $37^{\circ} \mathrm{C}$. The particles were then centrifuged (4200 g, $7 \mathrm{~min}$ for CAPs; $1500 \mathrm{~g}$, 3 min for CSPs) and washed three times with DPBS buffer to obtain the hPCcoated CAPs or CSPs. These hPC-coated particles were used directly for cell-association and proteomic experiments or further washed with different buffers or ultrapure water for characterization.

Characterization of Particles. After formation of hPCs, the CAPs and CSPs were isolated and characterized by the following methods. Differential interference contrast (DIC) and fluorescence microscopy images were taken with an inverted Olympus IX71 microscope. TEM images were acquired on a FEI cryo Tecnai Spirit instrument, operating at $120 \mathrm{kV}$. Prior to TEM analysis, samples $(1 \mu \mathrm{L})$ were dropped onto Formvarcoated copper grids and allowed to air-dry. Microelectrophoresis experiments (Zetasizer Nano ZS, Malvern Instruments) were used to determine the $\zeta$-potential of the particles with or without hPCs in phosphate buffer (pH 7.4, 5 mM).

Sodium Dodecyl Sulfate-Polyacrylamide Gel Electrophoresis (SDS-PAGE). The separation of proteins in the PC by using SDS-PAGE followed a protocol established previously. ${ }^{8}$ For the analysis, $1 \times 10^{8}$ PMA CAPs or CSPs were used to generate the hPC-coated particles following the protocols described above. The adsorbed proteins were stripped from the particles by adding NuPAGE LDS sample loading buffer and 
heated at $70{ }^{\circ} \mathrm{C}$ for $10 \mathrm{~min}$. The eluted proteins were transferred to a new tube. The disulfide bonds in proteins were cleaved using a reducing agent (50 mM dithiothreitol) and heated at $70{ }^{\circ} \mathrm{C}$ for $10 \mathrm{~min}$. The samples were then loaded on the gel and run for 50 min at 200 V. Each gel included one lane of a standard molecular weight ladder. The gel image was processed with the software Quantity One (Version 4.6.9) from Bio-Rad to estimate the total intensity of individual bands.

Cell Culture. Human monocytic leukemia cell line THP-1 (American Type Culture Collection) cells were maintained in RPMI1640 media with the addition of 10\% (v/v) FBS at $37{ }^{\circ} \mathrm{C}$ in a $5 \% \mathrm{CO}_{2}$ humidified atmosphere. Macrophage-like THP-1 (dTHP-1) were differentiated from THP- 1 cells by the treatment with $200 \mathrm{nM}$ TPA for $48 \mathrm{~h}$ in the complete growth media. Human colorectal carcinoma cell line LoVo was purchased from Cell Bank Australia and cultured in F-12K media containing $10 \%(\mathrm{v} / \mathrm{v}) \mathrm{FBS}$ at $37^{\circ} \mathrm{C}$ in a 5\% $\mathrm{CO}_{2}$ humidified atmosphere and subcultured prior to confluence. Human cervix epithelial adenocarcinoma cell line HeLa (American Type Culture Collection) cells were maintained in DMEM media containing $10 \%(\mathrm{v} / \mathrm{v}) \mathrm{FBS}$ at $37^{\circ} \mathrm{C}$ in a $5 \% \mathrm{CO}_{2}$ humidified atmosphere and subcultured prior to confluence.

Cellular Association of Particles by Flow Cytometry. The cellular association of particles was quantified as described previously. ${ }^{8,19}$ Cells were incubated with particles at a density of $1 \times 10^{5}$ cells in $0.5 \mathrm{~mL}$ of serum-free medium containing $1 \times 10^{7}$ AF633labeled PMA CAPs or CSPs (with or without hPCs) in 24-well plates at $37^{\circ} \mathrm{C}, 5 \% \mathrm{CO}_{2}$ for $3 \mathrm{~h}$. After treatment for $3 \mathrm{~h}$, cells were then collected and washed with DPBS three times with centrifugation at $300 \mathrm{~g}$ for 5 min between washes. The cell pellet was resuspended in DPBS and analyzed by flow cytometry (Apogee Flow). Analysis was 
performed using FlowJo vX.0.6. Cells that displayed 638-Red (AF633) signals above 100 (with a PMT setting of 638-Red at 600) were identified as those associated with the particles. The data were presented as a percentage of cells associated with the particles.

Cellular Association of Particles by Fluorescence Deconvolution Microscopy. hPCcoated PMA CAPs or CSPs were prepared following the protocols described above. THP-1 or dTHP-1 cells were treated with AF633-labeled PMA CAPs or CSPs (with or without hPCs) at a particle-to-cell ratio of 100:1. The cells were then washed with DPBS three times to remove excess particles and fixed with 4\% paraformaldehyde for $20 \mathrm{~min}$ at $23^{\circ} \mathrm{C}$. The cell membrane was stained with WGA488 $\left(2.5 \mu \mathrm{g} \mathrm{mL} \mathrm{L}^{-1}\right)$ in DPBS at $23^{\circ} \mathrm{C}$ for 15 min. Optical sections of cell images were collected using a fluorescence deconvolution microscope (Delta Vision, Applied Precision). Images were processed with Imaris 6.3.1 software (Bitplane) and presented in maximum intensity projection unless otherwise noted.

Cytokine Assay. PMA CAPs or CSPs (with or without various hPCs) were added to THP-1 or dTHP-1 cells at a particle-to-cell ratio of 100:1 and incubated for $24 \mathrm{~h}$ at $37^{\circ} \mathrm{C}$, $5 \% \mathrm{CO}_{2}$ in complete growth media. For THP-1 cells, the supernatant was collected by centrifugation at $400 \mathrm{~g}$ for 5 min to remove cells and then $16000 \mathrm{~g}$ for 5 min to remove any remaining particles. For dTHP-1 cells, the supernatant was collected from the adherent cells and then centrifuged at $16000 \mathrm{~g}$ for 5 min to remove any remaining particles. Cytokine levels in the supernatant were determined using ELISA kits (96 assay) and measured on an Infinite ${ }^{\circledR} 200$ PRO Microplate Reader (Tecan Group Ltd., Männedorf, Switzerland) as per the manufacturers’ instructions. 
Apoptosis Assay. PMA CAPs or CSPs (with or without various hPCs) were added to THP-1 or dTHP-1 cells at a particle-to-cell ratio of 100:1 and incubated for $24 \mathrm{~h}$ at $37{ }^{\circ} \mathrm{C}$, $5 \% \mathrm{CO}_{2}$ in complete growth media. THP-1 cells were harvested by centrifugation at 400 $g$ for 5 min. dTHP-1 cells were detached using $0.25 \%$ Trypsin solution for 5 min at $37^{\circ} \mathrm{C}$, followed by centrifugation at $400 \mathrm{~g}$ for $5 \mathrm{~min}$. Cells were then resuspended in $1 \mathrm{~mL}$ of DPBS buffer containing $0.1 \mu \mathrm{M}$ YO-PRO ${ }^{\circledR}-1$ and $1 \mu \mathrm{g} \mathrm{mL} \mathrm{m}^{-1}$ propidium iodide (PI) and incubated on ice for 30 min before flow cytometry analysis (Apogee Flow). The fluorescence from YO-PRO®-1 was measured through the 488-Grn with a PMT setting at 500 and PI was measured through 552-Org with a PMT setting at 500. Small angle light scattering (SALS) and large angle light scattering (LALS) properties were used to acquire a total of 10000 cells and to gate out the cell debris. Apoptotic cells were identified as YO-PRO®-1 positive, and dead cells identified at PI positive using FlowJo vX.0.6 software.

\section{RESULTS AND DISCUSSION}

Fabrication of PMA Particles and Formation of PC. Multilayered PMA particles, including CAPs and CSPs, were synthesized as described previously through the LbL assembly technique and copper(I)-catalyzed azide-alkyne cycloaddition (CuAAC)

chemistry ('click' chemistry). ${ }^{2,3}$ The assembled PMA CAPs are $\sim 1 \mu \mathrm{m}$ in diameter at physiological conditions (Figures 1A and S1A). The CAPs and CSPs were then incubated with media containing proteins (FBS, HS, HP, or media from cell cultures) to form PCs. The resulting PC-particle complexes were separated from the media by centrifugation and washed with Dulbecco’s phosphate-buffered saline (DPBS) extensively. In this work, 
we treat the PC-particle complexes after extensive washing as the hPC-coated PMA CAPs and CSPs. Particles were well dispersed in aqueous solution without visible aggregation after hPC coating, as shown for CAPs and CSPs with hPC from HP and LoVo-conditioned media (Figures 1B,C and S1B,C). A protein coating could be observed by transmission electron microscopy (TEM) for both the unconditioned (HP) and cellconditioned (LoVo) hPC-coated CSPs (Figure S1). Once air-dried, the hPC-coated CAPs collapsed, showing folds and creases (Figure 1).

Analysis of hPC Effects on Particles. Protein Adsorption. To analyze the hPCs, proteins were subsequently eluted from the particle surface, separated by SDS-PAGE, and stained with Coomassie G-250. It was shown that exposing the particles to various environments led to both qualitative and quantitative changes in the hPCs (Figures 2A,B and S2). Protein-rich conditions (100\% FBS, HS and HP) led to hPCs of at least four times higher total amount of adsorbed proteins compared to those formed in in vitro cellconditioned environments ( 10\% FBS plus cell-secreted proteins) (Figure 2B). This finding is in accordance with our previous report where we analyzed the amount of adsorbed protein in hPCs from 10, 50 and 100\% HS, and found an increasing amount of protein with increasing content of HS. ${ }^{19}$ Similar to our previous report, ${ }^{8}$ the composition of hPCs was dependent on the properties of the particles (Figure 2A). However, a common feature in all hPCs was a number of proteins in the region within $40-65 \mathrm{kDa}$ (Figures 2A and S2), where albumin, the most dominant protein in serum, elutes. ${ }^{19}$ It is noteworthy to mention that the cell-conditioned medium differed among the investigated cell lines according to the recommended culture growth medium, respectively. Subtle differences in medium composition might additionally contribute to differences in the 
$\mathrm{hPC}^{34}$ highlighting the complexity of this process. Further experiments revealed that hPCs from FBS, HS, HP, HeLa-, LoVo-, and THP-1-conditioned media, were long-lived and could partially retain their original composition even after subsequent $24 \mathrm{~h}$ incubation in complete cell growth media (Figure S3). Especially for hPCs derived from HS and HP, SDS-PAGE showed two protein bands between 25 and $40 \mathrm{kDa}$, which are characteristic for the environment the protein corona was formed, and which did not exchange upon incubation in complete cell growth media. To qualitatively understand the hPCs derived from HS and HP we also performed mass spectrometry (MS). MS analysis resulted in over 150 proteins detectable in these hPCs and are listed in Table S1.

Surface Charge. We next evaluated the effect of hPCs from different conditioned and unconditioned environments on the particle surface charge by performing a $\zeta$-potential analysis of the particles. In general, as reported previously, ${ }^{8}$ the adsorption of proteins led to "neutralization" of the particle surface charge for both the CAPs and CSPs (Figures 2C and S4); that is a decrease in surface charge. The decrease in surface charge was similar for all cell-conditioned PCs and less compared to the control of the same FBS amount (10\%). Notably, among all conditioned hPCs, the LoVo cell-conditioned hPC was the only one that had a negligible impact on the particle $\zeta$-potential. Further, the neutralizing effect of hPCs on particle surface charge was strongest for the commercialized serum environments (100\% FBS, HS, HP) where the protein concentrations are much higher than those in in vitro cell-conditioned environments. As discussed above hPCs from FBS, HS and HP contained significantly more proteins on the particles surface than hPCs from cell-conditioned media. This finding indicates that the neutralizing effect of hPCs on particle surface charge depends on the total amount of protein adsorbed on the particle 
surface for both the CAPs and CSPs (Figure 2B,C). A higher protein concentration may lead to higher surface coverage and different hPC composition, which results in different "neutralization levels” of the particle surface charge (Figure 2C).

Cell Association and Cellular Uptake. As PCs provide particles with different biological identities, we then investigated the cellular uptake of PMA CAPs and CSPs with hPCs from unconditioned and cell-conditioned environments. We studied cellular uptake using two immune cell lines (human monocytic leukemia cell line THP-1 and macrophage-like dTHP-1) and cancer cells (human cervix epithelial adenocarcinoma cell line HeLa). PMA CAPs and CSPs were fluorescently labeled with AF633, and counted using flow cytometry before and after the formation of hPCs. THP-1, dTHP-1 and HeLa cells were incubated with AF633-PMA particles in the absence or presence of hPCs for 3 $\mathrm{h}$ at $37^{\circ} \mathrm{C}$. The cells were then analyzed quantitatively by flow cytometry for surface bound and internalized particles (Figures 3 and S5). Particles with hPCs from the unconditioned environments (FBS, HS, HP) and three in vitro cell-conditioned environments (LoVo, HeLa, THP-1) were further qualitatively investigated by deconvolution fluorescence microscopy regarding their interaction with THP-1 and dTHP-1 cells (Figures S6 and S7). Generally, the presence of a hPC reduced the particlecell association and depended on the environment where the hPC was formed, on the cell phenotype (THP-1, dTHP-1 or HeLa cells) and on the properties of particles (CSPs or CAPs) (Figure 3). This observation is in agreement with other studies, in which cellular uptake of nanoparticles decreased due to the presence of serum proteins. ${ }^{17,18}$ Specifically, this inhibition effect on cellular uptake of particles by THP-1 and HeLa was stronger, resulting in a lower particle-cell association to these cells than to dTHP-1 cells (Figures 3 
and S5). This is in agreement with our previous study, which revealed that the PC played different roles in particle interactions with dTHP- 1 and THP- 1 cells, due to the presence of phagocytosis receptors on dTHP-1 cell surfaces. ${ }^{3}$ Cellular uptake was inhibited by hPCs to a similar extent toward THP-1 and HeLa cells, however, the uptake of PC-free particle was much higher for THP-1 than for HeLa cells suggesting different uptake mechanisms of phagocytes (THP-1) and cancer cells (HeLa) (Figure S5). Comparing the two particle systems, hPCs showed a stronger inhibition on capsule-cell association than on core-shell particle-cell association (Figures 3 and S5). This may be because of the difference in rigidity and sedimentation effects between CAPs and CSPs due to the absence or presence of the template. ${ }^{32}$ Interestingly, the LoVo cell-conditioned hPC inhibited the cellular uptake of both the CAPs and the CSPs by both immune cell lines (Figure 3). This finding suggests the possibility of tuning the composition of PCs from a certain source to reduce the uptake of particles by different immune cells and is relevant to achieve longer circulation times of particles. For that reason we chose the LoVo cellconditioned environment together with HeLa and THP-1 (which have been widely used for in vitro studies) cell-conditioned environments as representatives of human cancer and immune cell-conditioned environments for further particle-immune cell interactions. 

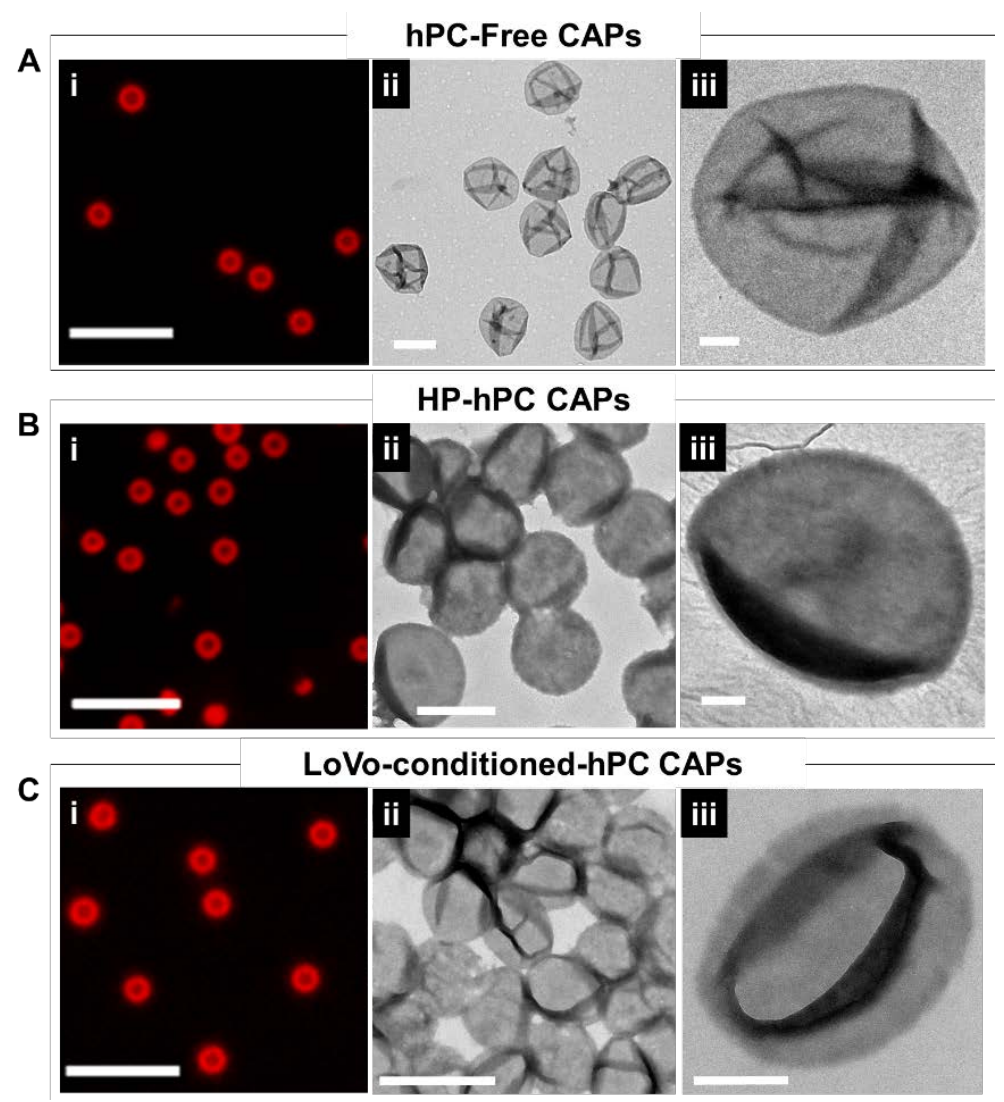

Figure 1. Fluorescence microscopy (i) and TEM (ii and iii) images of Alexa Fluor 633 hydrazide (AF633)-labeled (red) PMA CAPs without hPC (hPC-Free CAPs, A), or with hPC derived from HP (HP-hPC CAPs, B) and from LoVo cell-conditioned media (LoVoconditioned-hPC CAPs, C). (ii) Lower-resolution and (iii) higher-resolution TEM images of CAPs from the same samples. Scale bars are $5 \mu \mathrm{m}$ in (i), $1 \mu \mathrm{m}$ in (ii), and $200 \mathrm{~nm}$ in (iii). 

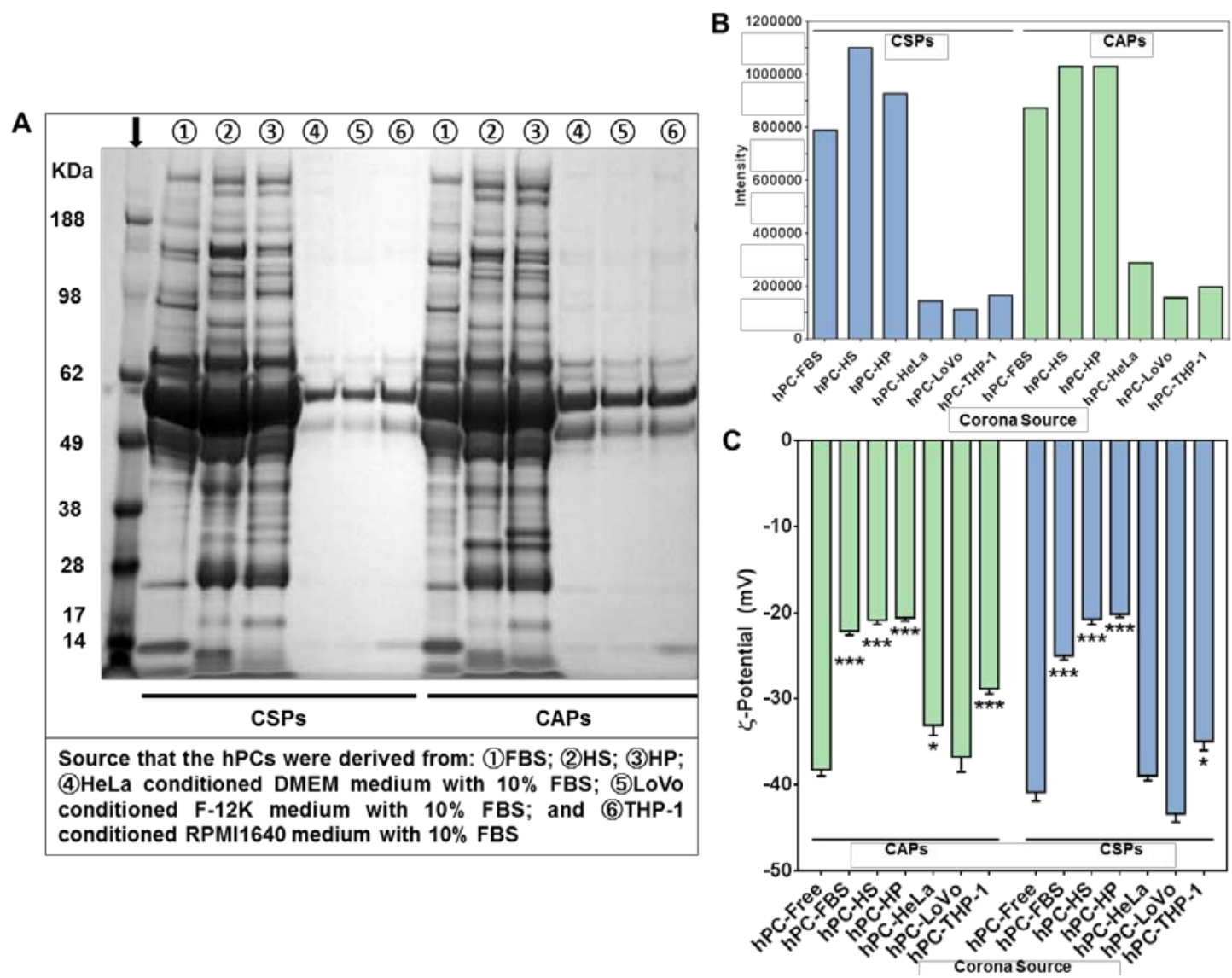

Figure 2. A) SDS-PAGE images of separated corona proteins derived from various environments on CSPs and CAPs. Reference bands associated with a particular molecular weight are displayed in the image (as indicated by the arrow). Numbers above the image indicate the source from which the hPCs were derived from. B) Total intensity of each lane, indicating the total amount of protein in the gel (A), analyzed using the 1-D analysis software Quantity One (Version 4.6.9) from Bio-Rad. C) ל-potential of PMA CAPs or CSPs with or without PCs derived from various environments. * $p<0.05$, *** $p<0.001$ versus the column of 'hPC-Free' (one-way ANOVA Dunnett's multiple comparison test). 

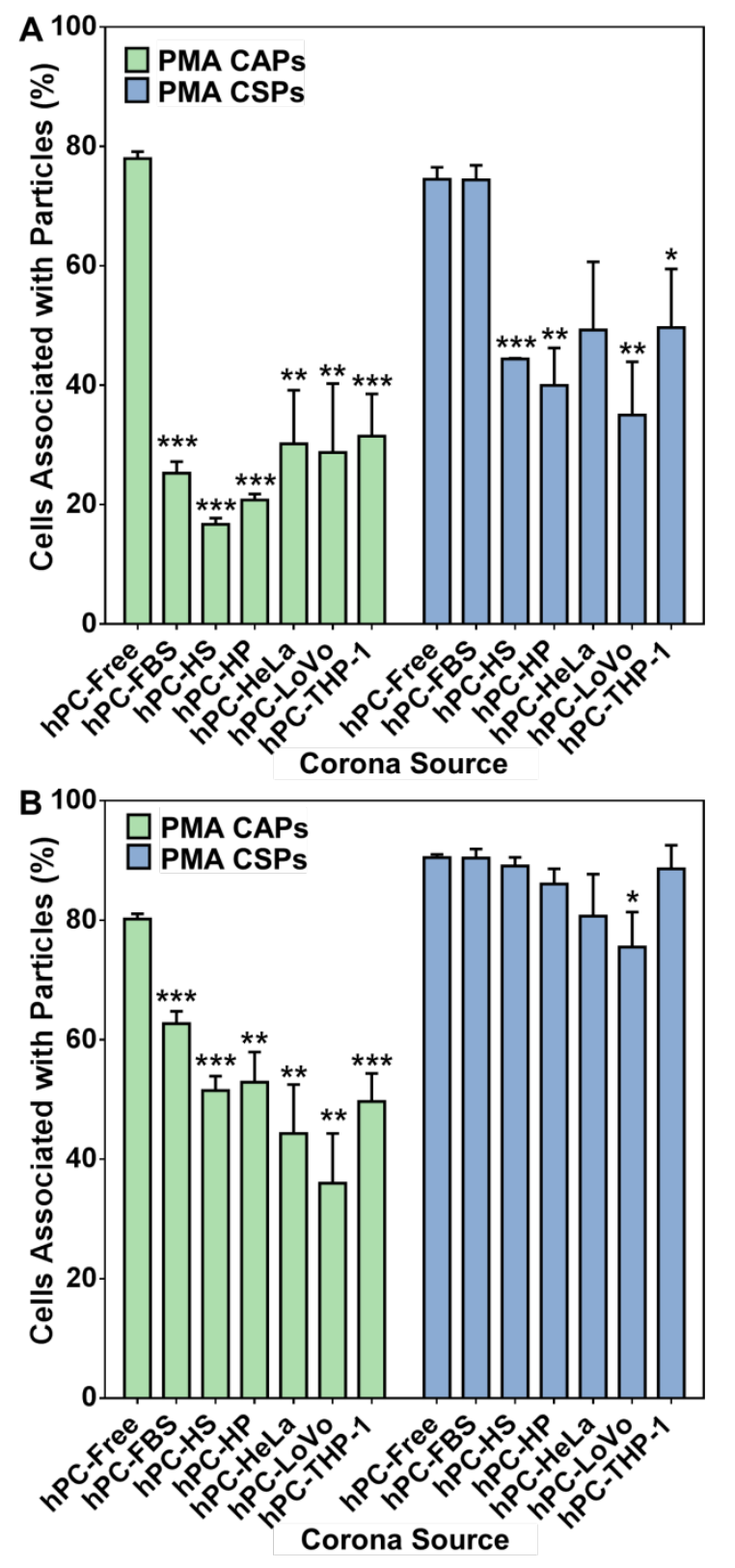

Figure 3. Cell association of PMA CAPs (green bars) or CSPs (blue bars) coated with different hPCs with THP-1 (A) and dTHP-1 (B) cells, as measured by flow cytometry. Data are shown as the mean \pm standard error of at least four independent experiments, with at least 10000 cells analyzed in each experiment. * $p<0.05$, ** $p<0.01$, *** $p<$ 0.001, versus the column of 'hPC-Free' (one-way ANOVA Dunnett's multiple comparison test). 
hPC Effects on Particle-Immune Cell Interactions. Cytokine Secretion. To investigate the immune responses induced by hPC-PMA particles, we compared bare particles, particles with hPC from a static, unconditioned environment, and particles with hPC from a dynamic, cell-conditioned environment. We performed experiments on immune responses in serum-free medium to minimize cross-effects from FBS. However, cell-conditioning was performed in the presence of FBS to mimic a protein-rich physiological environment. We first conducted a cytokine assay by measuring cytokine secretion levels after particle-cell interactions. Both THP-1 and dTHP-1 cells were treated with particles with or without hPCs for $24 \mathrm{~h}$ at $37^{\circ} \mathrm{C}, 5 \% \mathrm{CO}_{2}$. Subsequently, the respective supernatants were collected, and the levels of induced cytokines were determined using an enzyme-linked immunosorbent assay (ELISA). The secretion of 10 cytokines (Interleukin(IL)-1 $\beta$, IL-4, IL-6, IL-8, IL-10, IL-12, interferon(IFN)- $\gamma$, tumor necrosis factor (TNF)- $\alpha$, granulocyte-macrophage colony stimulating factor (GM-CSF), and monocyte chemoattractant protein-1 (MCP-1) by both THP-1 and dTHP-1 cells was evaluated using ELISA kits, respectively. Statistically significant changes in cytokine induction were observed in both cell lines after treatment with various hPC-PMA particles compared to untreated cells as well as to cells treated with hPC-free particles (Figures 4 and S8-S10). Both increased and reduced cytokine secretion levels were detected upon incubation with different hPC-coated particles. In general, the cytokine secretion of dTHP-1 cells was more readily affected by the presence of particles compared with THP-1 cells. For dTHP-1 cells, the levels of TNF- $\alpha$, IL-1 $\beta$, MCP-1, and IFN- $\gamma$ were most drastically influenced. For example, FBS-hPC-PMA CSPs induced a four-fold TNF- $\alpha$ secretion of dTHP-1 cells compared to the untreated dTHP-1 cells, and a 
1.3 fold TNF- $\alpha$ secretion compared to cells treated with hPC-free particles (Figure S9A). In contrast, the corresponding capsule system, FBS-hPC-PMA CAPs, led to a four-fold reduction of the TNF- $\alpha$ secretion by dTHP- 1 cells compared to untreated cells and to a 2.5 fold reduction compared to cells treated with hPC-free particles (Figure S9). Statistically significant differences in cytokine secretion were also found for IL- $1 \beta$ and MCP-1 where PMA CAPs with hPCs from different environments followed a reverse trend in cytokine induction. For IL-1 $\beta$ secretion, hPCs from FBS, HP and LoVoconditioned media significantly increased cytokine levels compared to untreated and hPC-free treated cells, while hPCs from HS, HeLa- and THP-1-conditioned media significantly lowered IL-1 $\beta$ levels. The opposite effect was observed for MCP-1 secretion; higher levels after treatment with particles of a hPC from HS, HeLa- and THP-1conditioned media, and decreased levels for hPCs from FBS, HP and LoVo-conditioned media. Comparing the effect of hPC-coated particles on cytokine secretion between the THP-1 and dTHP-1 cells, IL-8 levels were most striking. IL-8 levels in dTHP-1 cells were more than 20 times higher than in THP-1 cells and were only negligibly affected upon incubation with the various particle systems, while nearly all hPC-coated CAP and CSP systems induced statistically significant differences in IL-8 levels compared to untreated and hPC-free treated cells.

Taken together, the data demonstrate there is the potential in modulating cytokine secretion levels by exploiting subtle variations in hPCs when derived from a certain environment. In particular, the different responses of THP-1 and dTHP-1 cells when incubated with particles with hPCs formed either in an unconditioned or in an in vitro 
cell-conditioned environment highlight the possibility to fine-tune a particle system toward a specific cytokine induction or reduction of cytokine levels.

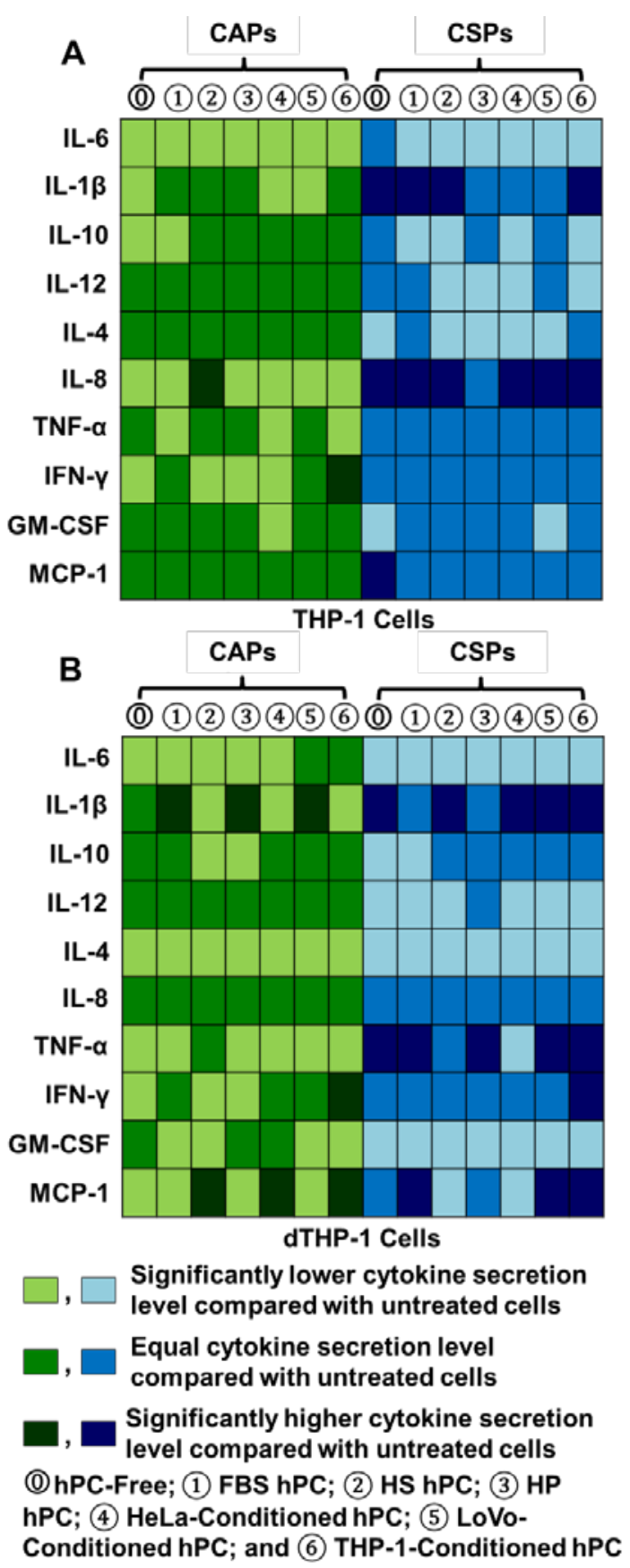

Figure 4. Heat maps showing the relative pro-inflammatory cytokine secretion levels by THP-1 (A) and dTHP-1 (B) cells. Cells were treated by CAPs (green) or CSPs (blue) coated with hPCs derived from various environments (as indicated by the numbers above 
the maps). The relative cytokine secretion levels are relative to those secreted by untreated cells (original data are shown in Figures S8 and S9).

Cell Death and Apoptosis. To evaluate the toxicity of hPC-coated particles to immune cells, YO-PRO ${ }^{\circledR}-1$ was used as a measure of early apoptosis, and the nucleic acid dye propidium iodide (PI) was used as the indicator of cell death. For THP-1 cells, PMA CAPs induced both apoptosis and cell death/necrosis (Figure 5A,B). In contrast, PMA CSPs did not change the THP-1 cell apoptosis level (Figure 5C), while PMA CSPs coated with HS, HP or LoVo cell-conditioned hPCs even led to a significant decrease of the THP-1 cell death levels (Figure 5D). Different findings were made for dTHP-1 cells: PMA CAPs showed less influence on both cell apoptosis and cell death/necrosis than PMA CSPs (Figure 5E-F). More specifically, only capsules with hPCs from cellconditioned environments and HP showed statistically significantly higher cell death/necrosis levels. In contrast, all core-shell particles with hPCs from unconditioned and cell-conditioned environments induced apoptosis as well as cell death. Combined, these results indicate that both the biological identities and synthetic properties of particles influence the cytotoxicity on immune cells. Importantly, among the different systems investigated we identified particles that either increased cytotoxicity and levels of apoptosis or lowered cell death levels, highlighting again the potential of hPCs as a means of influencing particle-immune cell interactions. 

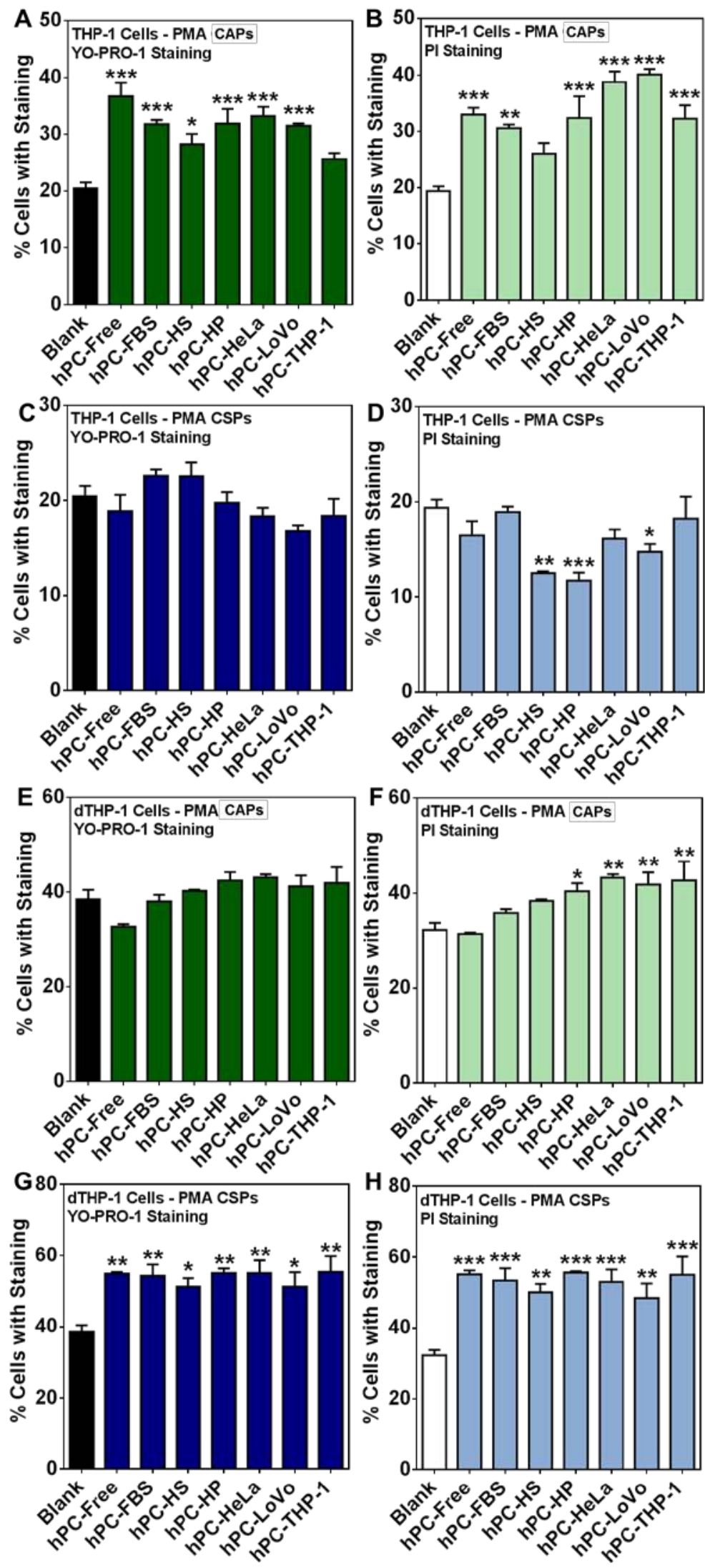
Figure 5. Early apoptosis and cell death, as measured by flow cytometry. THP-1 (A-D) and dTHP-1 (E-H) cells were treated by CAPs (A, B, E, F) or CSPs (C, D, G, H) with hPCs derived from various environments at $37{ }^{\circ} \mathrm{C}, 5 \% \mathrm{CO}_{2}$ for $24 \mathrm{~h}$. The cells were then stained with YO-PRO®-1 (A, C, E, G) to measure early apoptosis and propidium iodide (PI; B, D, F, H) to measure cell death. Data are shown as the mean \pm standard error of at least four independent experiments, with at least 10000 cells analyzed in each experiment. ${ }^{*} p<0.05,{ }^{* *} p<0.01,{ }^{* *} p<0.001$, versus the column of 'Blank' (indicating untreated cells) (one-way ANOVA Dunnett's multiple comparison test).

\section{CONCLUSIONS}

In this study, we demonstrated that hPCs from different unconditioned and cellconditioned environments influence the particle properties, affect particle-cell interactions, and induce immune responses differently. In the process of cell-conditioning, cells internalize nutrients from and secrete molecules into the surrounding, thereby generating a highly dynamic environment. It was shown earlier that this 'noncommercialized' composition affects the biological identity of a particle different to a static environment and induced AuNP aggregation. ${ }^{27}$ We observed well-dispersed particles upon PC formation in both unconditioned and in vitro cell-conditioned environments. However, particles varied in surface charge, amount of adsorbed proteins, and cellular uptake by phagocytes (THP-1, dTHP-1) and cancer cells (HeLa). In particular, the decreased uptake of particles with a hPC from LoVo-conditioned medium by phagocytes is an important finding for designing particles for enhanced circulation. Similarly, particles with PC coatings from different static or dynamic environments either 
increased or lowered cytokine levels. The induction of specific cytokine secretion is promising for vaccine delivery, while certain types of PCs might be used to reduce the inflammatory cytokine secretion and cell apoptosis, which, as a result, may help reduce the toxicity or side-effects of the particles. On the whole, our study shows that subtle differences in PCs offer specific coatings for surface functionalization, which presents an alternative avenue to engineer particles to influence bio-nano interactions.

\section{ASSOCIATED CONTENT}

Supporting Information. Additional experimental details, Figures S1-S10, and Table S1. This material is available free of charge via the Internet at http://pubs.acs.org.

\section{AUTHOR INFORMATION}

\section{Corresponding Author}

*E-mail: fcaruso@unimelb.edu.au

\section{Notes}

The authors declare no competing financial interest.

\section{Author Contributions}

The manuscript was written through contributions of all authors. All authors have given approval to the final version of the manuscript.

\section{ACKNOWLEDGMENT}

This research was conducted and funded by the Australian Research Council (ARC) Centre of Excellence in Convergent Bio-Nano Science and Technology (project number 
CE140100036). This work was also supported by the ARC under the Australian Laureate Fellowship (F.C., FL120100030). Q.D. and J.G. acknowledge funding from the University of Melbourne through Melbourne International Research Scholarships. This work was performed in part at the Materials Characterisation and Fabrication Platform (MCFP) at the University of Melbourne and the Victorian Node of the Australian National Fabrication Facility (ANFF).

\section{REFERENCES}

(1) Monopoli, M. P.; Åberg, C.; Salvati, A.; Dawson, K. A. Nat. Nanotechnol. 2012, 7, 779-786.

(2) Walkey, C. D.; Chan, W. C. W. Chem. Soc. Rev. 2012, 41, 2780-2799.

(3) Yan, Y.; Gause, K. T.; Kamphuis, M. M. J.; Ang, C.-S.; O'Brien-Simpson, N. M.; Lenzo, J. C.; Reynolds, E. C.; Nice, E. C.; Caruso, F. ACS Nano 2013, 7, 10960-10970.

(4) Cedervall, T.; Lynch, I.; Foy, M.; Berggård, T.; Donnelly, S. C.; Cagney, G.; Linse, S.; Dawson, K. A. Angew. Chem. Int. Ed. 2007, 46, 5754-5756.

(5) Cedervall, T.; Lynch, I.; Lindman, S.; Berggård, T.; Thulin, E.; Nilsson, H.; Dawson, K. A.; Linse, S. Proc. Nat. Acad. Sci. U.S.A 2007, 104, 2050-2055.

(6) Dell'Orco, D.; Lundqvist, M.; Oslakovic, C.; Cedervall, T.; Linse, S. PLOS One 2010, 5 .

(7) Röcker, C.; Pötzl, M.; Zhang, F.; Parak, W. J.; Nienhaus, G. U. Nat. Nanotechnol. 2009, 4, 577-580.

(8) Dai, Q.; Yan, Y.; Ang, C.-S.; Kempe, K.; Kamphuis, M. M. J.; Dodds, S. J.; Caruso, F. ACS Nano 2015, 2876-2885.

(9) Lundqvist, M.; Stigler, J.; Elia, G.; Lynch, I.; Cedervall, T.; Dawson, K. A. Proc. Nat. Acad. Sci. U.S.A 2008, 105, 14265-14270.

(10) Tenzer, S.; Docter, D.; Rosfa, S.; Wlodarski, A.; Kuharev, J.; Rekik, A.; Knauer, S. K.; Bantz, C.; Nawroth, T.; Bier, C.; Sirirattanapan, J.; Mann, W.; Treuel, L.; Zellner, R.; Maskos, M.; Schild, H.; Stauber, R. H. ACS Nano 2011, 5, 7155-7167.

(11) Walkey, C. D.; Olsen, J. B.; Guo, H. B.; Emili, A.; Chan, W. C. W. J. Am. Chem. Soc. 2012, 134, 2139-2147.

(12) Hühn, D.; Kantner, K.; Geidel, C.; Brandholt, S.; De Cock, I.; Soenen, S. J. H.; Gil, P. R.; Montenegro, J. M.; Braeckmans, K.; Müllen, K.; Nienhaus, G. U.; Klapper, M.; Parak, W. J. ACS Nano 2013, 7, 3253-3263.

(13) Casals, E.; Pfaller, T.; Duschl, A.; Oostingh, G. J.; Puntes, V. ACS Nano 2010, 4, 3623-3632.

(14) Tenzer, S.; Docter, D.; Kuharev, J.; Musyanovych, A.; Fetz, V.; Hecht, R.; Schlenk, F.; Fischer, D.; Kiouptsi, K.; Reinhardt, C.; Landfester, K.; Schild, H.; Maskos, M.; Knauer, S. K.; Stauber, R. H. Nat. Nanotechnol. 2013, 8, 772-781. 
(15) Lundqvist, M.; Stigler, J.; Cedervall, T.; Berggård, T.; Flanagan, M. B.; Lynch, I.; Elia, G.; Dawson, K. ACS Nano 2011, 5, 7503-7509.

(16) Palchetti, S.; Digiacomo, L.; Pozzi, D.; Peruzzi, G.; Micarelli, E.; Mahmoudi, M.; Caracciolo, G. Nanoscale 2016, 8, 12755-12763.

(17) Lesniak, A.; Fenaroli, F.; Monopoli, M. R.; Åberg, C.; Dawson, K. A.; Salvati, A. ACS Nano 2012, 6, 5845-5857.

(18) Lesniak, A.; Salvati, A.; Santos-Martinez, M. J.; Radomski, M. W.; Dawson, K. A.; Åberg, C. J. Am. Chem. Soc. 2013, 135, 1438-1444.

(19) Dai, Q.; Yan, Y.; Guo, J.; Björnmalm, M.; Cui, J.; Sun, H.; Caruso, F. ACS Macro Lett. 2015, 1259-1263.

(20) Salvati, A.; Pitek, A. S.; Monopoli, M. P.; Prapainop, K.; Bombelli, F. B.; Hristov, D. R.; Kelly, P. M.; Åberg, C.; Mahon, E.; Dawson, K. A. Nat. Nanotechnol. 2013, 8, 137-143.

(21) Zarschler, K.; Prapainop, K.; Mahon, E.; Rocks, L.; Bramini, M.; Kelly, P. M.; Stephan, H.; Dawson, K. A. Nanoscale 2014, 6, 6046-6056.

(22) Lunov, O.; Syrovets, T.; Loos, C.; Beil, J.; Delecher, M.; Tron, K.; Nienhaus, G. U.; Musyanovych, A.; Mailänder, V.; Landfester, K.; Simmet, T. ACS Nano 2011, 5, 1657-1669.

(23) Deng, Z. J.; Liang, M. T.; Monteiro, M.; Toth, I.; Minchin, R. F. Nat. Nanotechnol. 2011, 6, 39-44.

(24) Zhang, D. M.; Neumann, O.; Wang, H.; Yuwono, V. M.; Barhoumi, A.; Perham, M.; Hartgerink, J. D.; Wittung-Stafshede, P.; Halas, N. J. Nano Lett. 2009, 9, 666-671.

(25) Kulasingam, V.; Diamandis, E. P. Mol. Cell. Proteomics 2007, 6, 1997-2011.

(26) Skalnikova, H.; Motlik, J.; Gadher, S. J.; Kovarova, H. Proteomics 2011, 11, 691708.

(27) Albanese, A.; Walkey, C. D.; Olsen, J. B.; Guo, H. B.; Emili, A.; Chan, W. C. W. ACS Nano 2014, 8, 5515-5526.

(28) Zolnik, B. S.; González-Fernández, A.; Sadrieh, N.; Dobrovolskaia, M. A. Endocrinology 2010, 151, 458-465.

(29) Wang, Y.; Yan, Y.; Cui, J.; Hosta-Rigau, L.; Heath, J. K.; Nice, E. C.; Caruso, F. Adv. Mater. 2010, 22, 4293-4297.

(30) Sexton, A.; Whitney, P. G.; Chong, S. F.; Zelikin, A. N.; Johnston, A. P. R.; De Rose, R.; Brooks, A. G.; Caruso, F.; Kent, S. J. ACS Nano 2009, 3, 3391-3400.

(31) Hosta-Rigau, L.; Shimoni, O.; Städler, B.; Caruso, F. Small 2013, 9, 3573-3583.

(32) Cui, J.; De Rose, R.; Alt, K.; Alcantara, S.; Paterson, B. M.; Liang, K.; Hu, M.; Richardson, J. J.; Yan, Y.; Jeffery, C. M.; Price, R. I.; Peter, K.; Hagemeyer, C. E.; Donnelly, P. S.; Kent, S. J.; Caruso, F. ACS Nano 2015, 9, 1571-1580.

(33) Kinnane, C. R.; Such, G. K.; Antequera-Garcia, G.; Yan, Y.; Dodds, S. J.; LizMarzan, L. M.; Caruso, F. Biomacromolecules 2009, 10, 2839-2846.

(34) Maiorano, G.; Sabella, S.; Sorce, B.; Brunetti, V.; Malvindi, M. A.; Cingolani, R.; Pompa, P. P. ACS Nano 2010, 4, 7481-7491. 
Table of Contents graphic

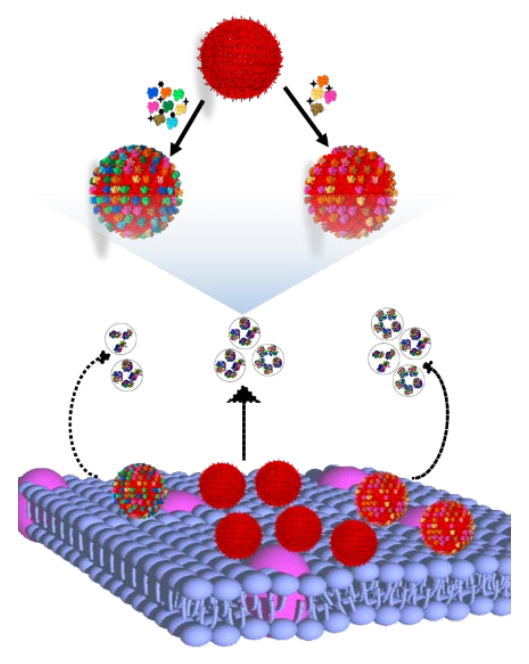

